\title{
What is the relationship between post-traumatic stress disorder, extreme appraisals of internal states and symptoms of bipolar disorder? \\ DOI:
}

$10.1017 / \mathrm{S} 1352465819000572$

\section{Document Version}

Final published version

Link to publication record in Manchester Research Explorer

Citation for published version (APA):

Frost, L., Tai, S., Varese, F., \& Mansell, W. (2020). What is the relationship between post-traumatic stress disorder, extreme appraisals of internal states and symptoms of bipolar disorder? Behavioural \& Cognitive Psychotherapy. https://doi.org/10.1017/S1352465819000572

Published in:

Behavioural \& Cognitive Psychotherapy

\section{Citing this paper}

Please note that where the full-text provided on Manchester Research Explorer is the Author Accepted Manuscript or Proof version this may differ from the final Published version. If citing, it is advised that you check and use the publisher's definitive version.

\section{General rights}

Copyright and moral rights for the publications made accessible in the Research Explorer are retained by the authors and/or other copyright owners and it is a condition of accessing publications that users recognise and abide by the legal requirements associated with these rights.

\section{Takedown policy}

If you believe that this document breaches copyright please refer to the University of Manchester's Takedown Procedures [http://man.ac.uk/04Y6Bo] or contact uml.scholarlycommunications@manchester.ac.uk providing relevant details, so we can investigate your claim.

\section{OPEN ACCESS}




\title{
What is the relationship between post-traumatic stress disorder, extreme appraisals of internal state and symptoms in bipolar disorder?
}

\author{
Laura Frost ${ }^{1, \star}$, Warren Mansell ${ }^{1}$, Filippo Varese ${ }^{1,2}$ and Sara Tai ${ }^{1}$ \\ ${ }^{1}$ School of Health Sciences, Division of Psychology and Mental Health, Faculty of Biology Medicine and Health, Manchester \\ Academic Health Science Centre, University of Manchester, 2nd Floor, Zochonis Building, Brunswick Street, Manchester \\ M13 9PL, UK and ${ }^{2}$ Complex Trauma and Resilience Research Unit, Greater Manchester Mental Health NHS Foundation \\ Trust, Manchester, UK \\ *Corresponding author. Email: laura.frost@student.staff.ac.uk
}

(Received 16 March 2019; revised 19 July 2019; accepted 21 August 2019; first published online 22 October 2019)

\begin{abstract}
Background: It is important to understand the factors associated with more severe mood symptoms in bipolar disorder. The integrative cognitive model of bipolar disorder proposes that extreme appraisals of changes to internal states maintain and exacerbate mood symptoms.

Aims: The current study aimed to investigate if post-traumatic stress disorder (PTSD) is related to current depressive and manic bipolar symptoms, and whether this relationship is mediated by appraisals of internal state.

Method: Participants with bipolar disorder $(n=82)$ from a randomized controlled trial of cognitive therapy for bipolar disorder (the TEAMS trial) completed self-reported questionnaires assessing appraisals of internal state, generalized anxiety symptoms, and self-reported and observer-rated depressive and manic symptoms. Clinical interviews assessed PTSD co-morbidity.

Results: Participants with bipolar and co-morbid PTSD $(n=27)$ had higher depressive symptoms and more conflicting appraisals than those without PTSD. Regression analyses found PTSD to be associated with depressive symptoms but not manic symptoms. Conflicting appraisals were found to be associated only with manic symptoms meaning that the planned mediation analysis could not be completed.

Conclusions: Findings provide partial support for the integrative cognitive model of bipolar disorder and highlight the need for transdiagnostic treatments in bipolar disorder due to the prevalence and impact of trauma and co-morbidity. Working on trauma experiences in therapy may impact on depressive symptoms for those with bipolar disorder and co-morbid PTSD.
\end{abstract}

Keywords: appraisals; bipolar disorder; integrative cognitive model; post-traumatic stress disorder (PTSD); trauma

\section{Introduction}

Bipolar disorder is often characterized as a severe and persisting disorder whereby people episodically experience periods of mania or hypomania and depression (Grande et al., 2016). Definitions state that more than $1 \%$ of the world population meet a diagnosis of bipolar disorder (Merikangas et al., 2011). In order to inform both the prevention and treatment of bipolar disorder, it is important to understand the factors that lead to more severe symptoms and clinical impact. One such candidate is a history of psychological trauma (Etain et al., 2013; Neria et al., 2005). A recent meta-analysis found that bipolar disorder was associated with a higher prevalence of potentially traumatic experiences in childhood compared with non-psychiatric controls (Palmier-Claus et al., 2016). There is growing evidence that exposure to trauma is associated with a range of adverse 
clinical outcomes in bipolar disorder (e.g. Agnew-Blais and Danese, 2016). However, is unclear whether the relationship between trauma and bipolar disorder is explained by psychological mechanisms, such as cognitive styles, implicated in the vulnerability and maintenance of characteristic bipolar symptoms. The current study examined the prevalence of post-traumatic stress disorder (PTSD) in a sample of people diagnosed with bipolar spectrum disorders enrolled in a randomized controlled trial of cognitive therapy. The study specifically aimed to test whether individuals with co-morbid PTSD, compared with those without, reported more extreme positive and negative appraisals of internal states; and whether these appraisals mediated the relationship between PTSD and bipolar disorder symptoms.

Many individuals with severe mental health difficulties, such as schizophrenia spectrum disorders and mood disorders, have experienced traumatic events. Within samples of people experiencing serious mental health difficulties, lifetime rates of reported trauma range from 49 to $100 \%$ (Grubaugh et al., 2011; Meade et al., 2009), with the rate of co-morbid PTSD being estimated in the range of 19-53\% (Cusack et al., 2006; Grubaugh et al., 2011; Mueser et al., 2002). Between 4 and $40 \%$ of people diagnosed with bipolar disorder also meet diagnostic criteria for PTSD (Cerimele et al., 2017; Hernandez et al., 2013; Merikangas et al., 2007; Otto et al., 2004). This compares with estimates of $8 \%$ in the general US population (American Psychiatric Association, 2000). PTSD is characterized by a constellation of symptoms including intrusive memories, dissociative reactions, alterations in mood, cognitions, arousal and reactivity (American Psychiatric Association, 2013). People diagnosed with bipolar disorder and co-morbid PTSD, compared with those without co-morbid PTSD, have a higher frequency of suicide attempts (Quarantini et al., 2010; Simon et al., 2004), a higher number of manic/hypomanic episodes (Passos et al., 2016), poorer quality of life (Otto et al., 2006; Passos et al., 2016; Quarantini et al., 2010), higher inter-episode depressive symptoms (Maguire et al., 2008) and higher rates of functional impairment (Passos et al., 2016). One study demonstrated that those with PTSD and bipolar disorder score higher for current mania, but no significant differences were found regarding current depressive symptoms (Quarantini et al., 2010). Little is currently known about the psychological mechanisms that may mediate the relationship between bipolar disorder and PTSD.

Research has shown that for people diagnosed with bipolar disorder, co-morbid anxiety disorders are highly prevalent with negative consequences and the negative impact increases with multiple anxiety disorders (Otto et al., 2006). Therefore, it is important to disentangle the impact of anxiety in bipolar disorders from the role of PTSD (Otto et al., 2006).

The integrative cognitive model of bipolar disorder and mood swings (Mansell et al., 2007) provides some potential cognitive mechanisms for the associations between PTSD and the symptoms of bipolar disorder. It proposes that central to the development of symptoms of bipolar disorder are multiple conflicting and personalized appraisals of changes to internal states. Appraisals are often goal related and can simultaneously be both positive (e.g. 'When I feel activated I will excel in whatever I'm doing') and negative (e.g. 'My activated mood means I'm going to have a breakdown') (Fisk et al., 2015). Behavioural responses to shifts in internal states are dependent upon the nature of the current appraisal occupying awareness. Individuals may attempt to suppress or enhance these internal states using counter-productive strategies, such as ascent behaviours (actions that increase activation, e.g. risk taking) or descent behaviours (actions that decrease activation, e.g. social withdrawal). The Hypomanic Attitudes and Positive Predictions Inventory (HAPPI) was developed to assess the extreme, conflicting appraisals of internal states at the heart of the model (Mansell, 2006). Research demonstrated that high scores on the HAPPI can distinguish bipolar disorder from healthy controls (Mansell, 2006), remitted unipolar depression (Alatiq et al., 2010; Mansell et al., 2011) and non-clinical controls with and without previous hypomanic experiences (Mansell et al., 2011). This provides evidence for the existence of appraisals that are unique to bipolar disorder, as opposed to being a feature of all mood disorders. Further evidence demonstrates 
that these appraisals are not merely a consequence of bipolar disorder; negative appraisals of high mood states can predict mood symptoms over the following month (Dodd et al., 2011b).

Vulnerability to bipolar disorder is proposed to be highest when internal states are appraised in extreme and conflicting ways. Kelly et al. (2011) found that individuals were most likely to have bipolar disorder when an activated state was endorsed both very positively and very negatively at the same time. No studies have tested whether these appraisals are associated with more severe current bipolar symptoms or the presence of PTSD. This might be expected given that conflict concerning trauma has also been proposed to be responsible for maintaining distress across severe mental health problems (Carey et al., 2014). Furthermore, it is important to explore whether the extreme appraisals that can predict mood symptoms (Dodd et al., 2011b) may be operating on the internal states involved in chronic traumatization (e.g. high arousal, emotionally activating memories).

The current study aimed to build on existing evidence by comparing appraisals in those with and without co-morbid PTSD in bipolar disorder. Specifically, we hypothesized that those with PTSD would have higher levels of current depressive and manic symptoms, and report more extreme positive and negative appraisals of internal states, compared with those without PTSD. Further, we aimed to test whether appraisals mediated the relationship between PTSD and bipolar disorder symptoms.

\section{Overall aims/research question}

The study examined the following hypotheses:

(1) Service users with bipolar and co-morbid PTSD have higher levels of manic and depressive symptoms than those without co-morbid PTSD.

(2) Service users with bipolar and co-morbid PTSD have higher positive appraisals, negative appraisals and conflicting appraisals than those without co-morbid PTSD.

(3) Across the whole sample, the interaction between positive and negative appraisals will be related to more severe manic and depressive symptoms.

(4) Appraisals of mood states mediate the relationship between PTSD and bipolar disorder symptoms.

\section{Method}

\section{Participants}

The current study is a secondary analysis of baseline data from the 'Think Effectively About Mood Swings' (TEAMS; Mansell et al., 2014) randomized control trial. Participants with a DSM-IV diagnosis of bipolar I or II disorder were recruited from NHS Trusts in the North West of England. Participants were deemed eligible if they met the following inclusion criteria: (i) having a DSM-IV diagnosis of bipolar I or II, characterized by a past DSM-IV hypomania of 2 days or more and a major depressive episode, (ii) were aged 16 or over, and (iii) had a score of at least 15 on the Beck Depression Inventory-II (BDI-II; Beck et al., 1996) for two out of the six weeks prior to the study. Participants meeting the following criteria were excluded: (i) DSM-IV diagnosis of non-affective psychotic disorder, (ii) current mania or mixed episode according to DSM-IV, (iii) a primary DSM-IV substance use disorder, (iv) moderate to severe learning disability, (v) organic impairment accounting for mental health problem, or (vi) non-English speaking.

The sample characteristics are summarized in Table 1; chi-square tests demonstrated no significant differences between those with and without PTSD for gender, diagnosis or ethnicity. A $t$-test showed no significant difference in age between those with PTSD and those without PTSD. Forty-three participants met the criteria for bipolar I disorder and 39 met the 
Table 1. Demographic and clinical characteristics of the sample

\begin{tabular}{lccc}
\hline & PTSD $=27(33 \%)$ & No-PTSD $=54(67 \%)$ & $p$ \\
\hline Age (years) & $44.78(S D=9.10)$ & $44.28(S D=13.13)$ & .864 \\
Gender & & & .064 \\
Male & $5(6 \%)$ & $21(26 \%)$ & \\
Female & $22(27 \%)$ & $33(41 \%)$ & .064 \\
Diagnosis & & & \\
Bipolar I & $15(19 \%)$ & $27(33 \%)$ & .361 \\
Bipolar II & $12(15 \%)$ & $27(33 \%)$ & \\
Ethnicity & & & \\
White British & $23(28 \%)$ & $48(59 \%)$ & \\
Other* & $4(5 \%)$ & $6(7 \%)$ & \\
Number of anxiety co-morbidities & & & \\
Total & 66 & $12(15 \%)$ & \\
0 & $1(1 \%)$ & $25(31 \%)$ & \\
1 & $8(10 \%)$ & $9(11 \%)$ & \\
2 & $8(10 \%)$ & $4(5 \%)$ & \\
3 & $2(2 \%)$ & $4(5 \%)$ & \\
4 & $4(5 \%)$ & $0(0 \%)$ & \\
5 & $4(5 \%)$ & $33(42 \%)$ & \\
Anti-depressants & $15(19 \%)$ & $34(44 \%)$ \\
Anti-psychotics & $15(19 \%)$ & $1(1 \%)$ \\
Benzodiazepines/hypnotics & $4(5 \%)$ & $23(29 \%)$ \\
Anti-convulsant & $10(13 \%)$ & $15(19 \%)$ \\
Lithium & $3(4 \%)$ & $5(6 \%)$ & \\
No medication & $2(3 \%)$ & & \\
\hline
\end{tabular}

Other ethnicities include Pakistani, other Asian, African, White Irish, other White, White and Asian, other mixed, other ethnic group. Medication information is missing for four participants. PTSD information is missing for one participant.

criteria for bipolar II disorder. Twenty-seven participants met the diagnostic criteria for PTSD. This equated to PTSD being present in 33\% of the sample, $19 \%$ of men and $40 \%$ of women.

\section{Measures}

The Structured Clinical Interview for Axis I DSM-IV Disorders (SCID-I; First et al., 2002)

The SCID-I was used to establish participants' psychiatric diagnoses. To verify that participants met the study selection criteria, the screening module, Module A (Mood Episodes) and the Psychosis screener of the SCID-I were administered. The PTSD module was used to identify participants meeting diagnostic criteria for post-traumatic stress.

\section{Demographic questionnaire}

Participants were asked a series of questions regarding clinical and demographic characteristics. Information was acquired regarding gender, age and current medications.

The Beck Depression Inventory-II (BDI-II; Beck et al., 1996)

The BDI-II was used to assess depression severity over the previous fortnight. Participants were asked to respond to 21 items, each consisting of four statements describing increasing levels of severity of specific depressive symptoms (rated from 0 to 3). Higher BDI-II total scores indicate higher depression severity. The BDI-II has demonstrated good psychometric properties, including high internal consistency and good test-retest reliability in clinical and non-clinical samples (Beck et al., 1996). Cronbach's alpha was .92 in the current sample.

The Hamilton Depression Rating Scale (HAM-D; Hamilton, 1960)

The HAM-D is a 17-item interview-based measure of depression that covers a range of behavioural, affective and biological symptoms. Each item is scored on either a 3- or a 5-point scale. These scores are then summed to provide a total depression severity score. Reportedly, 
the inter-rater reliability is between .70 and .96 and the test-retest reliability is between .65 and .96 (Kobak, 2003). Cronbach's alpha was .82 in the current sample.

The Internal States Scale (ISS; Bauer et al., 1991)

The ISS is a 16-item scale assessing depressive and manic symptoms over the previous 24 hours. The ISS consists of four subscales: Perceived conflict ( 5 items; e.g. 'Today I feel argumentative'), Activation (5 items; e.g. 'Today I feel impulsive'), Depression (2 items; e.g. 'Today I feel depressed') and Well-being (3 items; e.g. 'Today I actually feel great inside'). Participants rate to what degree they experienced the described internal state on a 0 (rarely) to 100 (much of the time) scale. High scores indicate greater symptom severity for all subscales except the Well-being subscale. Internal consistencies for the subscales ranged from Cronbach's $\alpha=.92$ for Depression to .81 for Perceived conflict (Bauer et al., 1991). Perceived conflict was the only subscale included in the current analysis, for which Cronbach's alpha was .83 .

The Bech-Rafaelsen Mania Rating Scale (BMRS; Bech et al., 1978)

The BMRS is an observer-rated measure of manic symptoms across 11 domains: motor activity, verbal activity, flight of thoughts, voice/noise level, hostility, mood, self-esteem, contact, sleep, sexual interest and work. Each domain is assessed using statements rated on a scale of 0 (not present, normal function) to 4 (obviously present e.g. 'impossible to interrupt'), with higher total scores indicating greater manic symptom severity. The BMRS is reported to have high inter-observer reliability, acceptable external validity and good internal validity (Bech, 2002). Cronbach's alpha was .71 in the current sample.

The Generalised Anxiety Disorder-7 (GAD-7; Spitzer et al., 2006)

The GAD-7 is a 7-item self-report questionnaire used as a severity measure for generalized anxiety disorder symptoms over the previous 2 weeks. It is scored on a 4 -point Likert scale $(0=$ not at all, $1=$ several days, $2=$ more than half the days, $4=$ nearly every day). This scale has demonstrated both high levels of internal consistency $(\alpha=.89-.92)$ and test-retest reliability $(r=.83$; Löwe et al., 2008; Spitzer et al., 2006). The current study found Cronbach's alpha to be 91 .

The Hypomanic Attitudes and Positive Predictions Inventory (HAPPI; Dodd et al., 2010)

The extended 61-item version of the HAPPI was used to measure extreme positive and negative appraisals of activated states over the previous week. Participants responded on a visual analogue scale ranging from 0 ('I don't believe this at all') to 100 ('I believe this completely'). Strong internal consistency has been found for the overall scale with Cronbach's alpha reported at .97 (Dodd et al., 2011b), which is supported by the current study which reported Cronbach's alpha at .96. The HAPPI provides a positive (e.g. 'When I feel excited, my fears and worries are no longer real') and negative appraisals subscale score (e.g. 'The better I feel about myself, the worse other people react towards me'). The positive and negative subscales were calculated by taking a mean score of the items. The mean scores from the positive and negative subscales were multiplied together to calculate the interaction term. In this study, Cronbach's alpha was .87 for the positive appraisals items, and .77 for the negative appraisal items.

\section{Procedure}

The study was approved by the London Queen Square Research Ethics Committee (REC reference 11/LO/1326). Participants were recruited through clinical staff and advertisements in community mental health teams, outpatient services, GP practices, research volunteer organizations and the voluntary sector. In addition, advertisements were placed on mental health charities' websites and 
Table 2. Correlational matrix using rank biserial (for analyses with PTSD status) and Spearman's correlations

\begin{tabular}{|c|c|c|c|c|c|c|c|c|}
\hline & PTSD & $\mathrm{BDI}$ & BMRS & HAM-D & ISS Conflict & GAD-7 & $\begin{array}{l}\text { Positive } \\
\text { appraisals }\end{array}$ & $\begin{array}{c}\text { Negative } \\
\text { appraisals }\end{array}$ \\
\hline BDI-II & $.39^{\star \star \star}$ & & & & & & & \\
\hline BMRS & .15 & .16 & & & & & & \\
\hline HAM-D & $.45^{\star \star \star}$ & $.67^{\star \star \star}$ & $.35^{\star \star \star}$ & & & & & \\
\hline ISS Conflict & .17 & $.38^{\star \star \star}$ & $.24^{\star}$ & $.26^{\star}$ & & & & \\
\hline GAD-7 & $.27^{\star}$ & $.72^{\star \star \star}$ & $.36^{\star \star \star}$ & $.54^{\star \star \star}$ & $.51^{\star \star \star}$ & & & \\
\hline Positive appraisals & $.22^{*}$ & .20 & $.29^{\star \star}$ & .17 & .20 & $.22^{\star}$ & & \\
\hline Negative appraisals & $.24^{\star}$ & .18 & .14 & .20 & $.39^{\star \star \star}$ & .21 & $.58^{\star \star \star}$ & \\
\hline Conflicting appraisals & $.23^{*}$ & .19 & $.23^{*}$ & .20 & $.36^{\star \star \star}$ & $.24^{\star}$ & $.83^{\star \star \star}$ & $.92^{\star \star \star}$ \\
\hline
\end{tabular}

Rank biserial correlations are reported for all correlations that include the PTSD variable; Spearman's correlations are reported for the remaining correlations. PTSD, post-traumatic stress disorder. BDI, Beck Depression Inventory; BMRS, Bech-Rafaelson Mania Scale; HAM-D, Hamilton Rating Scale for Depression; ISS, Internal States Scale; GAD-7, Generalized Anxiety Disorder-7. ${ }^{\star} p<.05 ;{ }^{\star \star} p<.01 ;{ }^{* \star \star} p<.001$.

local media to reach potential participants who were not currently accessing mental health services. Trained research assistants conducted initial screening interviews over the telephone or in person to ensure that interested individuals met inclusion criteria. For those who met the inclusion criteria, written informed consent was obtained and then baseline assessments were completed in person. For further information on the procedure, participant recruitment and additional measures included, please consult the study protocol for the TEAMS trial (Mansell et al., 2014).

\section{Data analysis}

Inspection of descriptive statistics, histograms and the Kolmogorov-Smirnov test were used to identify outliers and determine whether the data met assumptions for parametric analysis. As the majority of the variables did not meet the assumptions of normality, a non-parametric analysis approach was conducted. Rank biserial and Spearman's correlations were used to test associations between PTSD and bipolar disorder symptoms, positive appraisals, negative appraisals and conflicting appraisals. These analyses were followed up by a series of two-step hierarchical regression analyses to determine if PTSD predicted bipolar disorder symptoms while controlling for GAD-7 scores, and if PTSD predicted appraisals while controlling for GAD-7 scores. It has been advised that to detect a medium effect size when the desired statistical power is at the recommended level of 0.8 (Cohen, 1988) for two predictor variables, a sample size of 68 is required (Field, 2013), suggesting that the current analyses which included 82 participants, were appropriately powered. As no significant associations were found between PTSD and manic symptoms and appraisals and depressive symptoms, it was not appropriate to continue with the planned mediation analysis. This decision was additionally based upon concerns that the mediation analysis would be insufficiently powered (Fritz and MacKinnon, 2007). All analyses were conducted using SPSS (version 22). All statistical tests were two-tailed and used a significance level of 0.05 .

\section{Results}

No outliers were identified. Correlational analyses revealed robust associations between PTSD and the variables measuring depressive symptoms, the BDI-II and the HAM-D (Table 2). There were significant positive associations between PTSD and the GAD-7, positive appraisals, negative appraisals and conflicting appraisals. PTSD was not associated with the variables measuring mania symptoms, the BRMS or the ISS conflict. Positive appraisals were associated with the BRMS, negative appraisals were associated with the ISS conflict and conflicting appraisals were associated with both the BRMS and ISS conflict. Appraisals did not show any associations with the BDI-II or the HAM-D. The GAD-7 demonstrated robust associations with the BDI-II, HAM-D, BRMS and ISS conflict. 
Table 3. Summary of hierarchical regression analyses investigating if PTSD predicts current bipolar symptoms

\begin{tabular}{|c|c|c|c|}
\hline Variable & $\beta$ & $p$ & $s r^{2}$ \\
\hline \multicolumn{4}{|l|}{ BDI-II } \\
\hline \multicolumn{4}{|l|}{ Step 1} \\
\hline PTSD & .38 & $<.001$ & .147 \\
\hline \multicolumn{4}{|l|}{ Step 2} \\
\hline PTSD & .21 & .011 & .041 \\
\hline GAD-7 & .64 & $<.001$ & .38 \\
\hline \multicolumn{4}{|l|}{ HAM-D } \\
\hline \multicolumn{4}{|l|}{ Step 1} \\
\hline PTSD & .43 & $<.001$ & .188 \\
\hline \multicolumn{4}{|l|}{ Step 2} \\
\hline PTSD & .31 & .001 & .09 \\
\hline GAD-7 & .46 & $<.001$ & .193 \\
\hline \multicolumn{4}{|l|}{ BRMS } \\
\hline \multicolumn{4}{|l|}{ Step 1} \\
\hline PTSD & .12 & .307 & .013 \\
\hline \multicolumn{4}{|l|}{ Step 2} \\
\hline PTSD & .04 & .741 & .001 \\
\hline GAD-7 & .29 & .012 & .076 \\
\hline \multicolumn{4}{|l|}{ ISS Conflict } \\
\hline \multicolumn{4}{|l|}{ Step1 } \\
\hline PTSD & .17 & .130 & .029 \\
\hline \multicolumn{4}{|l|}{ Step 2} \\
\hline PTSD & .02 & .835 & .000 \\
\hline GAD-7 & .54 & .000 & .263 \\
\hline
\end{tabular}

PTSD, post-traumatic stress disorder; BDI-II, Beck Depression Inventory-II; BMRS, Bech-Rafaelson Mania Scale; HAM-D, Hamilton Rating Scale for Depression; ISS, Internal States Scale; GAD-7, Generalized Anxiety Disorder-7.

\section{Regression analyses}

A first hierarchical regression was carried out with the BDI-II as the dependent variable (Table 3 ). After including PTSD in the first step of the analysis $(F(1,79)=13.66, p=<.001)$, the adjusted $R^{2}=.14$, indicating that PTSD explained $14 \%$ of the variance in the BDI-II. The inclusion of the GAD-7 variable at step 2 improved the prediction of BDI-II scores $\left(R^{2}=.52, F(2,78)=43.60\right.$, significance of $R^{2}$ change, $\left.p=.001\right)$. In the final step of the analysis, PTSD survived as a significant predictor of BDI-II scores. GAD-7 scores also significantly contributed to the prediction of BDI-II scores. Inspection of the squared semi-partial correlations $\left(s r^{2}\right)$ indicated that PTSD uniquely explained approximately $4 \%$ of the observed variance in BDI-II scores and GAD-7 explained $38 \%$ of the variance.

A similar regression model was estimated with the HAM-D as the dependent variable (Table 3). After including PTSD in the first step of the analysis $(F(1,79)=18.38, p=<.001)$, the adjusted $R^{2}=.18$, indicating that PTSD explained $18 \%$ of the variance in the HAM-D. The inclusion of the GAD-7 variable at step 2 improved the prediction of the HAM-D scores $\left(F(2,78)=24.04, R^{2}=.37\right.$, significance of $R^{2}$ change, $\left.p=.001\right)$. In the final step of the analysis, PTSD survived as a significant predictor of HAM-D scores. GAD-7 scores also significantly contributed to the prediction of HAM-D scores. Inspection of the $s r^{2}$ indicated that PTSD uniquely explained approximately $9 \%$ of the observed variance in HAM-D scores and GAD-7 explained 19\% of the variance.

A third hierarchical regression was carried out with the BRMS as the dependent variable (Table 3 ). PTSD was included in the first step of the analysis but it could not reliably predict variance in the BRMS $\left(F(1,79)=1.06, p=.31, R^{2}\right.$ adjusted $\left.=.001\right)$. Entering the GAD-7 variable at step 2 improved the prediction of BRMS scores $\left(F(2,78)=3.84, R^{2}=.09\right.$, significance of $R^{2}$ change, $\left.p=.01\right)$. Inspection of the $s r^{2}$ indicated that GAD-7 uniquely explained approximately $8 \%$ of the observed variance in BRMS scores. 
Table 4. Summary of hierarchical regression analyses investigating if PTSD predicts appraisals of internal states

\begin{tabular}{llll}
\hline Variable & $\beta$ & $p$ & $s r^{2}$ \\
\hline $\begin{array}{l}\text { Positive appraisals } \\
\text { Step 1 }\end{array}$ & & & \\
PTSD & .23 & .042 & .051 \\
Step 2 & & .119 & .029 \\
$\quad$ PTSD & .18 & .114 & .03 \\
GAD-7 & .18 & & \\
Negative appraisals & & .053 & .047 \\
Step 1 & .22 & .16 & .023 \\
PTSD & .16 & .063 & .042 \\
Step 2 & .21 & & .063 \\
PTSD & & .024 & .037 \\
GAD-7 & .25 & .078 & .034 \\
Conflicting appraisals & & .09 & \\
Step 1 & .20 & .19 & \\
PTSD & .19 & \\
Step 2 & & & \\
PTSD & & & \\
GAD-7 & & & \\
\hline
\end{tabular}

PTSD, post-traumatic stress disorder; GAD-7, Generalized Anxiety Disorder-7.

A hierarchical regression was estimated with the ISS conflict as the dependent variable (Table 3). Again, PTSD was included in step 1 but it could not reliably predict variance in the ISS conflict variable $\left(F(1,78)=2.34, p=.13, R^{2}\right.$ adjusted $\left.=.02\right)$. The inclusion of the GAD-7 variable at step 2 improved the prediction of the ISS conflict scores $\left(F(2,77)=15.92, R^{2}=.29\right.$, significance of $R^{2}$ change, $\left.\mathrm{p}=.001\right)$. Inspection of the $s r^{2}$ indicated that GAD-7 uniquely explained approximately $26 \%$ of the observed variance in ISS conflict scores.

A hierarchical regression was conducted with positive appraisals as the dependent variable (Table 4). After including PTSD in the first step of the analysis $(F(1,79=4.26, p=.04)$, the adjusted $R^{2}=.04$ indicating that PTSD explained $4 \%$ of the variance in positive appraisals. The inclusion of GAD-7 in the second step did not reliably improve the prediction of positive appraisals $\left(F(2,78)=3.45, R^{2}=.08\right.$, significance of $R^{2}$ change, $\left.p=.114\right)$.

In a separate model with negative appraisals as the criterion variable (Table 4$)$, the inclusion of PTSD in the first step of the analysis did not reliably predict variance $(F(1,79)=3.86, p=.053$, adjusted $\left.R^{2}=.04\right)$. The inclusion of GAD-7 in the second step of the analysis did not reliably improve the prediction of variance in negative appraisals $\left(F(2,78)=3.77, R^{2}=.09\right.$, significance of $R^{2}$ change, $p=.06$ ).

In a final hierarchical regression, conflicting appraisals were used as the dependent variable. After including PTSD in the first step of the analysis, the adjusted $R^{2}=.05(F(1,79)=5.33$, $p=.02$ ), indicating that PTSD explained $5 \%$ of the variance in conflicting appraisals. The inclusion of GAD-7 in the second step did not reliably improve the prediction of conflicting appraisals $\left(R^{2}=.10, F(2,78)=4.19\right.$, significance of $R^{2}$ change, $\left.p=.09\right)$.

\section{Discussion}

The results from the current study demonstrated that people with bipolar disorder and co-morbid PTSD present with higher current depressive symptoms than those without co-morbid PTSD. No significant difference was found for mania symptoms as hypothesized. Those with co-morbid PTSD appraised their internal states in more conflicting ways than those without PTSD. Additionally, conflicting appraisals of internal states were positively associated with current mania symptoms. Due to a lack of associations found between PTSD and mania symptoms and appraisals and depressive symptoms, the planned mediation analysis was not conducted. 
Within the current sample, it was found that $33 \%$ of the sample had co-morbid PTSD, which was towards the top end of the range of prevalence of PTSD reported in bipolar disorder (4-40\%) in previous investigations (Cerimele et al., 2017). Proportionately, more females were found to have PTSD than men, supporting previous findings of women being nearly twice as likely to have PTSD (Baldassano et al., 2005). A co-morbid anxiety disorder was found to be present in $84 \%$ of the sample.

In partial support of the first hypothesis, those with bipolar disorder and co-morbid PTSD were found to have higher depressive symptoms, in line with previous research (Maguire et al., 2008). However, those with and without PTSD did not significantly differ on current manic symptoms, inconsistent with the findings of several previous investigations. For example, Quarantini et al. (2010) found those with co-morbid bipolar and PTSD to have higher mania symptoms but did not find significant differences for depressive symptoms. Meta-analytic studies have indicated that trauma exposure is associated with greater mania severity (Agnew-Blais and Danese, 2016).

In comparison with those without co-morbid PTSD, those with bipolar disorder and co-morbid PTSD were found to have significantly more conflicting appraisals and negative appraisals, whilst the difference for positive appraisals was just outside of significance. Across the sample, co-morbid PTSD was significantly positively associated with conflicting appraisals, positive appraisals and negative appraisals. Bipolar disorder with co-morbid PTSD was found to be a significant predictor of conflicting appraisals and positive appraisals but not negative appraisals. Interestingly, GAD did not significantly contribute to the regression models, suggesting that the relationship between PTSD and appraisals of internal state is unique and cannot be extended to anxiety disorders. This is an area which was previously untested but the results may be expected in the context of the integrative cognitive model (Mansell et al., 2007). The model proposes that past life events affect personal beliefs about the self and beliefs about internal states which in turn influence appraisals. One could hypothesize that a severely traumatic life event is likely to influence appraisals, especially if the trauma was experienced during a mood episode or considered to be a trigger for the development of bipolar disorder. Conflict concerning trauma has also been suggested to be responsible for maintaining distress within populations with severe mental health difficulties (Carey et al., 2014).

The relationship between appraisals of internal states and current bipolar disorder symptoms has not previously been assessed in a sample of participants with bipolar disorder. Correlational analyses revealed that conflicting appraisals were positively related to manic symptoms but no significant associations were found for depressive symptoms. Positive appraisals were positively related to scores on the Bech-Rafelson Mania Rating Scale (BMRS; Bech et al., 1978), whereas negative appraisals were related to scores on the perceived conflict subscale of the Internal States Scale (ISS; Bauer et al., 1991). These results were in partial support of the third hypothesis, but significant associations were not found for depressive symptoms as expected. Kelly et al. (2011) found positive and negative appraisals to be correlated with both depressive and activation symptoms. However, this study utilized a non-clinical sample of undergraduate students and purely used two subscales from the ISS (Bauer et al., 1991), while the current study included more extensive observer-rated assessment of current mood states. At the centre of the integrative cognitive model (Mansell et al., 2007) are appraisals of changes to internal states that are extreme, multiple and conflicting. Dependent on which appraisal is occupying awareness, individuals may engage in counter-productive behaviours in an attempt to exert control over the mood state. Supporting evidence for this has emerged from the finding that multiple and conflicting appraisals are positively related to higher manic symptoms, although no associations were found between appraisals and depressive symptoms as anticipated.

Symptoms of generalized anxiety were controlled for throughout the analysis and found to be significantly associated with PTSD, and depressive and manic symptoms. This supports the previous literature, which suggests that concurrent anxiety has a negative impact in bipolar disorder. This was an important addition to the current study as it isolated the role of PTSD from generalized anxiety symptoms. 


\section{Implications and future directions}

The current study has added to the literature consistently demonstrating that rates of PTSD are high within bipolar disorder populations and related to the severity of depressive symptoms. This suggests that for people with bipolar disorder and co-morbid PTSD, working on trauma experiences in therapy may impact on depressive symptoms. Furthermore, focusing in therapy on how individuals appraise their internal states, specifically identifying and working with any conflicting appraisals, could be a useful method to assist in managing hypomanic/manic mood states.

The current study was cross-sectional, meaning that cause and effect could not be determined. Previous research has provided preliminary evidence that in a bipolar disorder sample, the HAPPI (Dodd et al., 2010) can prospectively predict outcomes on the ISS (Bauer et al., 1991) and the Work and Social Adjustment Scale (Mundt et al., 2002) over a 4-week period, while controlling for current symptoms (Dodd et al., 2011b). To provide further evidence of the predictive power of the HAPPI (Dodd et al., 2010) and to expand on the current research, it could be investigated if the predictor variables from the current study can prospectively predict bipolar symptoms while controlling for current symptoms.

The ideal study attempting to establish mediation for the effects of trauma would include a measure of conflict around goals surrounding trauma (Carey et al., 2014) as opposed to only conflict between appraisals of activated mood states. Indeed, an ideal study testing the Carey et al. (2014) proposal need not be specific to patients with a diagnosis of bipolar disorder, but would include a wide range of mental health problems in people reporting past trauma.

\section{Limitations}

A number of factors should be considered when interpreting the findings of this study. The inclusion criteria specified that participants must be at least moderately depressed (to target in therapy), and the exclusion criteria specified that participants could not be in manic episodes. Therefore, when investigating current symptoms, proportionately more participants were in current depressive episodes in comparison with those displaying manic symptoms.

There is a wealth of literature focusing on the relationship between various forms of trauma and the impact on the clinical course of bipolar disorder. The current study measured trauma as a binary variable based upon PTSD being present or not present. This may be limiting as it did not allow for the analysis of the impact of different forms of trauma (for example, childhood sexual abuse $v s$ violent assault). Also, it did not capture traumas that may have been severe but where participants did not meet PTSD criteria. The inclusion of a lifetime traumatic events questionnaire would have been desirable. However, the benefit of only including people meeting diagnostic threshold for PTSD is that the study focuses exclusively on traumas causing individuals current difficulties that might have psychological mechanisms maintaining them.

Previous literature has demonstrated that multiple anxiety disorders can impact negatively upon the clinical course of bipolar disorder (Otto et al., 2006). The current study only controlled for symptoms of generalized anxiety within the analysis which may not have captured the full range of difficulties associated with other anxiety disorders (e.g. social anxiety, panic disorder).

\section{Conclusion}

This study offers preliminary evidence for a relationship between PTSD and appraisals of internal states in bipolar disorder. The results also support previous literature to suggest that co-morbid PTSD can have a negative impact on the course of bipolar disorder. The study offers partial support for the integrative cognitive model (Mansell et al., 2007). This model has previously demonstrated its utility in explaining mood swings in patients with bipolar disorder who were not selected for their interest in seeking psychological therapy (Alatiq et al., 2010; Dodd et al., 2011a; Kelly et al., 2011; Mansell, 2006; Mansell et al., 2011). The mixed findings 
here may have emerged from the high rate of co-morbidities, suggesting that the model may have encountered difficulties in accounting for all of the complexities of this anxious and traumatized population. This study highlights the prevalence and detrimental effects of trauma in bipolar disorder and emphasizes the importance of taking a transdiagnostic perspective (Mansell, 2016).

Acknowledgements. Thank you to those who gave their time to participate in this study. Thank you to the following research assistants who contributed to the recruitment, assessment and risk management of participants: Sarah Alsawy, Anna Ridding, Savas Akgonul, Lydia Pearson, Alex Clark and Kate Lawrence.

Conflicts of interest. Laura Frost, Dr Warren Mansell, Dr Filippo Varese and Dr Sara Tai have no conflicts of interest with respect to this publication.

Funding. This paper presents independent research funded by the National Institute for Health Research (NIHR) under its Research for Patient Benefit (RfPB) Programme (Grant Reference Number PB-PG-0110-21087). The views expressed are those of the author(s) and not necessarily those of the NIHR or the Department of Health and Social Care.

Ethical statements. The study was approved by the London Queen Square Research Ethics Committee (REC reference 11/LO/1326). All authors have abided by the Ethical Principles of Psychologists and Code of Conduct as set out by the APA.

\section{References}

Agnew-Blais, J., \& Danese, A. (2016). Childhood maltreatment and unfavourable clinical outcomes in bipolar disorder: a systematic review and meta-analysis. The Lancet Psychiatry, 3, 342-349. doi: 10.1016/S2215-0366(15)00544-1

Alatiq, Y., Crane, C., Williams, J. M. G., \& Goodwin, G. M. (2010). Dysfunctional beliefs in bipolar disorder: hypomanic vs. depressive attitudes. Journal of Affective Disorders, 122, 294-300. doi: 10.1016/j.jad.2009.08.021

American Psychiatric Association (2000). Diagnostic and Statistical Manual of Mental Disorders, 4th text revision edn. Washington, DC, USA: American Psychiatric Association.

American Psychiatric Association. (2013). Diagnostic and Statistical Manual of Mental Disorders: DSM-5. Washington, DC, USA: American Psychiatric Association.

Baldassano, C. F., Marangell, L. B., Gyulai, L., Nassir Ghaemi, S., Joffe, H., Kim, D. R., . . \& Cohen, L. S. (2005). Gender differences in bipolar disorder: retrospective data from the first 500 STEP-BD participants. Bipolar Disorders, 7, 465-470. doi: 10.1111/j.1399-5618.2005.00237.x

Bauer, M. S., Crits-Christoph, P., Ball, W. A., Dewees, E., McAllister, T., Alahi, P., Cacciola, J., \& Whybrow, P. C. (1991). Independent assessment of manic and depressive symptoms by self-rating: scale characteristics and implications for the study of mania. Archives of General Psychiatry, 48, 807-812. doi: 10.1001/archpsyc.1991.01810330031005

Bech, P. (2002). The Bech-Rafaelsen Mania Scale in clinical trials of therapy for bipolar disorder: a 20-year review of its use as an outcome measure. CNS Drugs, 16, 47-63. doi: 10.2165/00023210-200216010-00004

Bech, P., Rafaelsen, O. J., Kramp, P., \& Bolwig, T. G. (1978). The Mania Rating Scale: scale construction and inter-observer agreement. Neuropharmacology, 17, 430-431. doi: 10.1016/0028-3908(78)90022-9

Beck, A. T., Steer, R. A., \& Brown, G. K. (1996). Beck Depression Inventory-II. San Antonio, 78, 490-498.

Carey, T. A., Mansell, W., Tai, S. J., \& Turkington, D. (2014). Conflicted control systems: the neural architecture of trauma. Lancet Psychiatry, 1, 316-318. doi: 10.1016/S2215-0366(14)70306-2

Cerimele, J. M., Bauer, A. M., Fortney, J. C., \& Bauer, M. S. (2017). Patients with co-occurring bipolar disorder and posttraumatic stress disorder: a rapid review of the literature. Journal of Clinical Psychiatry, 78, e506-514. doi: 10.4088/JCP.16r10897

Cohen, J. (1988). Statistical Power Analysis for the Social Sciences. Hillsdale, NJ, USA: Erlbaum.

Cusack, K. J., Grubaugh, A. L., Knapp, R. G. \& Frueh, B. C. (2006). Unrecognized trauma and PTSD among public health consumers with chronic and severe mental illness. Community Mental Health Journal, 42, 487-500. doi: 10.1007/s10597006-9049-4

Dodd, A., Mansell, W., Bentall, R., \& Tai, S. (2011a). Do extreme beliefs about internal states predict mood swings in an analogue sample? Cognitive Therapy \& Research, 35, 497-504. doi: 10.1007/s10608-010-9342-y

Dodd, A. L., Mansell, W., Morrison, A. P., \& Tai, S. (2011b). Extreme appraisals of internal states and bipolar symptoms: the Hypomanic Attitudes and Positive Predictions Inventory. Psychological Assessment, 23, 635. doi: 10.1037/a0022972

Dodd, A. L., Mansell, W., Sadhnani, V., Morrison, A. P., \& Tai, S. (2010). Principal component analysis of the Hypomanic Attitudes and Positive Predications Inventory and associations with measures of personality, cognitive style and analogue symptoms in a student sample. Behavioural and Cognitive Psychotherapy, 38, 15-33. doi: 10.1017/ S1352465809990476 
Etain, B., Aas, M., Andreassen, O. A., Lorentzen, S., Dieset, I., Gard, S., Kahn, J. P., Bellivier, F., Leboyer, M., Melle, I., \& Henry, C. (2013). Childhood trauma is associated with severe clinical characteristics of bipolar disorder. Journal of Clinical Psychiatry, 74, 991-998. doi: 10.4088/JCP.13m08353

Field, A. (2013). Discovering Statistics Using IBM SPSS Statistics. Los Angeles and London: Sage.

First, M. B., Spitzer, R. L., Gibbon, M., \& Williams, J. B. (2002). Structured Clinical Interview for DSM-IV-TR Axis I Disorders, Research Version, Patient Edition With Psychotic Screen (SCID-I/P W/ PSY SCREEN). New York, NY, USA: Biometrics Research, New York State Psychiatric Institute.

Fisk, C., Dodd, A. L., \& Collins, A. (2015.) Response styles, bipolar risk, and mood in students: the Behaviours Checklist. Psychology and Psychotherapy: Theory, Research and Practice, 88, 412-426. doi: 10.1111/papt.12052

Fritz, M. S., \& MacKinnon, D. P. (2007). Required sample size to detect the mediated effect. Psychological Science, 18, 233-239. doi: 10.1111/j.1467-9280.2007.01882.x

Grande, I., Berk, M., Birmaher, B., \& Vieta, E. (2016). Bipolar disorder. The Lancet, 387, 1561-1572. doi: 10.1016/S01406736(15)00241-X

Grubaugh, A. L., Zinzow, H. M., Paul, L., Egede, L. E., \& Frueh, B.C. (2011). Trauma exposure and posttraumatic stress disorder in adults with severe mental illness: a critical review. Clinical Psychology Review, 31, 883-899. doi: 10.1016/ j.cpr.2011.04.003

Hamilton, M. (1960). A rating scale for depression. Journal of Neurology, Neurosurgery, and Psychiatry, 23, 56-62. doi: 10.1136/jnnp.23.1.56

Hernandez, J. M., Cordova, M. J., Ruzek, J., Reiser, R., Gwizdowski, I. S., Suppes, T., \& Ostacher, M. J. (2013). Presentation and prevalence of PTSD in a bipolar disorder population: a STEP-BD examination. Journal of Affective Disorders, 150, 450-455. doi: 10.1016/j.jad.2013.04.038

Kelly, R. E., Mansell, W., Wood, A. M., Alatiq, Y., Dodd, A., \& Searson, R. (2011). Extreme positive and negative appraisals of activated states interact to discriminate bipolar disorder from unipolar depression and non-clinical controls. Journal of Affective Disorders, 134, 438-443. doi: 10.1016/j.jad.2011.05.042

Kobak, K. A. (2003). The Hamilton Depression Scale. In Hersen, M., Hilsenroth, M. J., \& Segal, D. L. (eds), Comprehensive Handbook of Psychological Assessment: Personality Assessment. New York, USA: John Wiley \& Son.

Löwe, B., Decker, O., Müller, S., Brähler, E., Schellberg, D., Herzog, W., \& Herzberg, P. Y. (2008). Validation and standardization of the Generalized Anxiety Disorder Screener (GAD-7) in the general population. Medical Care, 46, 266-274. doi: 10.1097/MLR.0b013e318160d093

Maguire, C., McCusker, C. G., Meenagh, C., Mulholland, C., \& Shannon, C. (2008). Effects of trauma on bipolar disorder: the mediational role of interpersonal difficulties and alcohol dependence. Bipolar Disorders, 10, 293-302. doi: 10.1111/ j.1399-5618.2007.00504.x

Mansell, W. (2006). The Hypomanic Attitudes and Positive Predictions Inventory (HAPPI): a pilot study to select cognitions that are elevated in individuals with bipolar disorder compared to non-clinical controls. Behavioural and Cognitive Psychotherapy, 34, 467-476. doi: 10.1017/S1352465806003109

Mansell, W. (2016). A positive mood cannot be too positive: how to utilise positive and negative clinical psychology in bipolar disorder. In Positive Clinical Psychology: An Integrative Approach to Studying and Improving Well-Being. New York, USA: Wiley.

Mansell, W., Morrison, A. P., Reid, G., Lowens, I., \& Tai, S. (2007). The interpretation of, and responses to, changes in internal states: an integrative cognitive model of mood swings and bipolar disorders. Behavioural and Cognitive Psychotherapy, 35, 515-539. doi: 10.1017/S1352465807003827

Mansell, W., Paszek, G., Seal, K., Pedley, R., Jones, S., Thomas, N., . . \& Dodd, A. (2011). Extreme appraisals of internal states in bipolar I disorder: a multiple control group study. Cognitive Therapy and Research, 35, 87-97. doi: 10.1007/s10608009-9287-1

Mansell, W., Tai, S. J., Clark, A., Akgonul, S., Dunn, G., Davies, L., Law, H., Morriss, R., Tinning, N., \& Morrison, A. P. (2014). A novel cognitive behaviour therapy for bipolar disorders (Think Effectively About Mood Swings or TEAMS): study protocol for a randomized controlled trial. Trials, 15, 405. doi: 10.1186/1745-6215-15-405

Meade, C. S., Kershaw, T. S., Hansen, N. B., \& Sikkema, K. J. (2009). Long-term correlates of childhood abuse among adults with severe mental illness: adult victimization, substance abuse, and HIV sexual risk behavior. AIDS and Behavior, 13, 207-216. doi: 10.1007/s10461-007-9326-4

Merikangas, K. R., Akiskal, H. S., Angst, J., Greenberg, P. E., Hirschfeld, R. M. A., Petukhova, M., \& Kessler, R. C. (2007). Lifetime and 12 month prevalence of bipolar disorder in the national comorbidity survey replication. Archives of General Psychiatry, 64, 543-552. doi: 10.1001/archpsyc.64.5.543

Merikangas, K. R., Jin, R., He, J. P., Kessler, R. C., Lee, S., Sampson, N. A., . . \& Zarkov, Z. (2011). Prevalence and correlates of bipolar spectrum disorder in the world mental health survey initiative. Archives of General Psychiatry, 68, 241-251. doi: 10.1001/archgenpsychiatry.2011.12

Mueser, K.T., Rosenberg, S.D., Goodman, L.A., \& Trumbetta, S.L. (2002). Trauma, PTSD, and the course of schizophrenia: an interactive model. Schizophrenia Research, 53, 123-143. doi: 10.1016/S0920-9964(01)00173-6 
Mundt, J. C., Marks, I. M., Shear, M. K., \& Griest, J. M. (2002). The Work and Social Adjustment Scale: a simple measure of impairment in functioning. British Journal of Psychiatry, 180, 461-464. doi: 10.1192/bjp.180.5.461

Neria, Y., Bromet, E. J., Carlson, G. A., \& Naz, B. (2005). Assaultive trauma and illness course in psychotic bipolar disorder: findings from the Suffolk county mental health project. Acta Psychiatrica Scandinavica, 111, 380-383. doi: 10.1111/j.16000447.2005.00530.x

Otto, M. W., Perlman, C. A., Wernicke, R., Reese, H. E., Bauer, M.S., \& Pollack, M. H. (2004). Posttraumatic stress disorder in patients with bipolar disorder: a review of prevalence, correlates, and treatment strategies. Bipolar Disorders, 6, 470-479. doi: 10.1111/j.1399-5618.2004.00151.x

Otto, M. W., Simon, N. M., Wisniewski, S. R., Miklowitz, D. J., Kogan, J. N., Reilly-Harrington, N. A., . . \& Weiss, R. D. (2006). Prospective 12-month course of bipolar disorder in out-patients with and without comorbid anxiety disorders. British Journal of Psychiatry, 189, 20-25. doi: 10.1192/bjp.bp.104.007773

Palmier-Claus, J. E., Berry, K., Bucci, S., Mansell, W., \& Varese, F. (2016). Relationship between childhood adversity and bipolar affective disorder: systematic review and meta-analysis. British Journal of Psychiatry, 209, 454-459. doi: 10.1192/ bjp.bp. 115.179655

Passos, I. C., Jansen, K., Cardoso, T. D. A., Colpo, G. D., Zeni, C. P., Quevedo, J., . . \& Kapczinski, F. (2016). Clinical outcomes associated with comorbid posttraumatic stress disorder among patients with bipolar disorder. Journal of Clinical Psychiatry, 77, 555-560. doi: 10.4088/JCP.15m09935

Quarantini, L. C., Miranda-Scippa, Â., Nery-Fernandes, F., Andrade-Nascimento, M., Galvão-de-Almeida, A., Guimarães, J. L., ... \& Post, R. M. (2010). The impact of comorbid posttraumatic stress disorder on bipolar disorder patients. Journal of Affective Disorders, 123, 71-76. doi: 10.1016/j.jad.2009.08.005

Simon, N. M., Otto, M. W., Wisniewski, S. R., Fossey, M., Sagduyu, K., Frank, E., . . \& Pollack, M. H. (2004). Anxiety disorder comorbidity in bipolar disorder patients: data from the first 500 participants in the Systematic Treatment Enhancement Program for Bipolar Disorder (STEP-BD). American Journal of Psychiatry, 161, 2222-2229. doi: 10.1176/appi.ajp.161.12.2222

Spitzer, R. L., Kroenke, K., Williams, J. B., \& Löwe, B. (2006). A brief measure for assessing generalized anxiety disorder: the GAD-7. Archives of Internal Medicine, 166, 1092-1097. doi: 10.1001/archinte.166.10.1092

Cite this article: Frost L, Mansell W, Varese F, and Tai S (2020). What is the relationship between post-traumatic stress disorder, extreme appraisals of internal state and symptoms in bipolar disorder? Behavioural and Cognitive Psychotherapy 48 , 103-115. https://doi.org/10.1017/S1352465819000572 